\title{
Moyamoya Syndrome Associated with Protein C and S Deficiency as a Neurological Complication in Children with Sickle Cell Disease
}

\author{
Yassien $\mathbf{M}^{1 *}$, El-Shanshory $\mathbf{M}^{2}$ and Asslan $\mathbf{M}^{3}$ \\ ${ }^{1}$ Department of Neurology, Tanta University, Egypt \\ ${ }^{2}$ Department of Pediatrics, Tanta University, Egypt \\ ${ }^{3}$ Department of Hematology, Tanta University, Egypt \\ *Corresponding author: Marwa Yassien, Department \\ of Neurology, Tanta University, Faculty of Medicine, \\ Egypt
}

Received: March 08, 2021; Accepted: March 18, 2021; Published: March 25, 2021

\begin{abstract}
Background: Sickle Cell Disease (SCD) is an autosomal recessive hemoglobinopathy containing mutant sickle cell Hemoglobin (HbS). Acute and chronic organ dysfunction, chronic hemolytic anemia, and recurrent painful episodes are the main features of sickle cell disease. There is a wide variety of neurological complications, including: headache, cognitive difficulties, seizures, visual loss, ischemic and hemorrhagic stroke, transient ischemic attacks, soft neurological signs coma, altered mental status and covert or silent infarction. Moyamoya is an uncommon cerebral vasculopathy that's also found in children with SCD.
\end{abstract}

Case Presentation: Here, we reported two children diagnosed with SCD who presented with headache, recurrent ischemic strokes, seizures and cognitive decline. They showed abnormalities on different neuroimaging including: CT and /or MRI, MRA and/or CT angiography, also transcranial color coded duplex, EEG and Stanford-Binet Intelligence scales-Fifth Edition. They also showed abnormal level of protein $\mathrm{C}$ and protein $\mathrm{S}$. They also had irregular blood transfusion and were diagnosed as Moyamoya syndrome.

Conclusion: According to our findings, Moyamoya syndrome was a rare complication of SCD in children, but should be considered with proper approach, diagnosis and management.

Keywords: SCD; Neurological complications; Moyamoya; Diagnosis

\section{Introduction}

Sickle Cell Disease (SCD) is an autosomal recessive hemoglobin disorder. It is a qualitative hemoglobinopathy resulting from a structural change in the sequence of amino acids on the beta globin chain of the hemoglobin molecule due to a point mutation. It is characterized by hemoglobin polymerization, erythrocyte stiffening, and subsequent vaso-occlusion [1,2].

The different sickle cell syndromes that result from distinct inheritance pattern of the sickle cell gene (beta S gene) are divided into sickle cell disease and sickle cell trait. Sickle cell disease is associated with chronic anemia and recurrent visceral pain. The sickle cell trait is largely asymptomatic [3]. The prevalence of SCD is $0.3 \%$ in Egypt, where the carrier rate varies from 9 to $22 \%$ [4].

Children with sickle cell disease, present with a wide variety of neurological syndromes, including ischemic and hemorrhagic stroke, transient ischemic attacks, soft neurological signs, seizures, headache, coma, visual loss, altered mental status, cognitive difficulties, and covert or 'silent' infarction [5].

Moyamoya is an uncommon cerebral vasculopathy, characterized by typical angiographic changes associated with subsequent clinical features. In moyamoya, steno-occlusive changes in the main cerebral arteries and decrease in the cerebral perfusion pressure, resulting in the formation of a fine network in neovascularization $[6,7]$.
These collaterals show evidence of stress related to increased flow, including the combination of the fragmented elastic lamina, thinned media in the vessel wall, and the presence of microaneurysms; these findings help to explain why some patients present with hemorrhage. Other Moyamoya related vessels are collapse, and their lumen thrombosis could cause ischemic symptoms $[8,9]$.

Moyamoya syndrome even found in sickle cell trait patients, but as a rare neurological complication, but it is found more in sickle cell disease patients $[10,11]$.

Children and adults with MM may have different clinical presentations; the symptoms and clinical course vary widely, ranging from being asymptomatic to manifesting as severe neurologic deficits. Children more commonly have ischemic events and may experience hemiparesis, monoparesis, sensory impairment, involuntary movements, headaches, dizziness, seizures, mental retardation, or persistent neurologic deficits. In adults, symptoms and signs are similar to those in children, but sudden onset intraventricular, subarachnoid, or intracerebral hemorrhage is more common [12].

\section{Case Presentation}

\section{Case 1}

A male child aged 9 years diagnosed at the age of 1 year with SCD with negative family history for SCD and positive consanguinity. He presented at first by recurrent attacks of episodic left temporal 


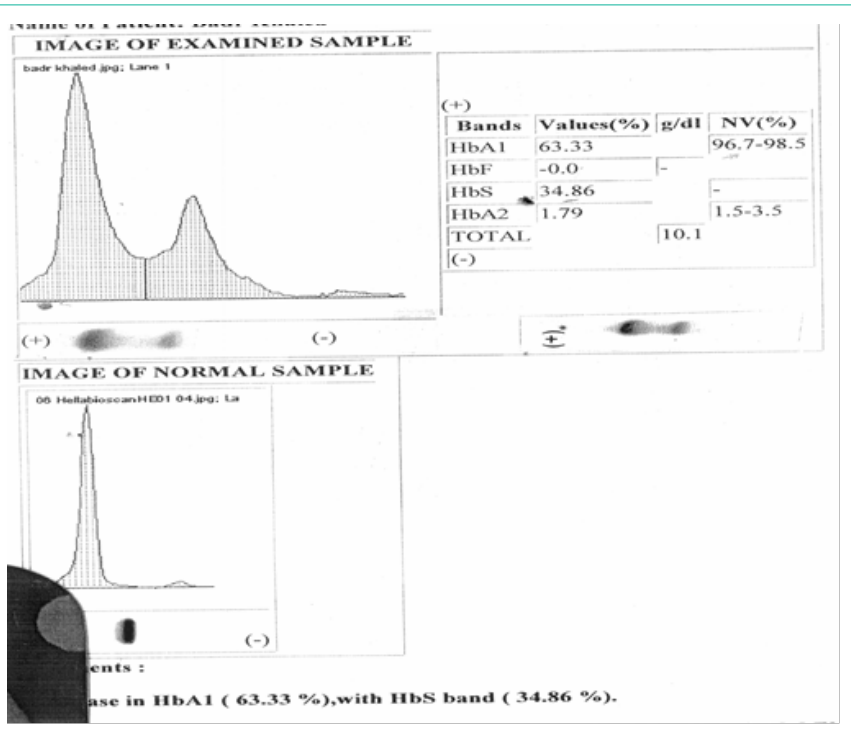

Figure (1a): Hb electrophoresis.
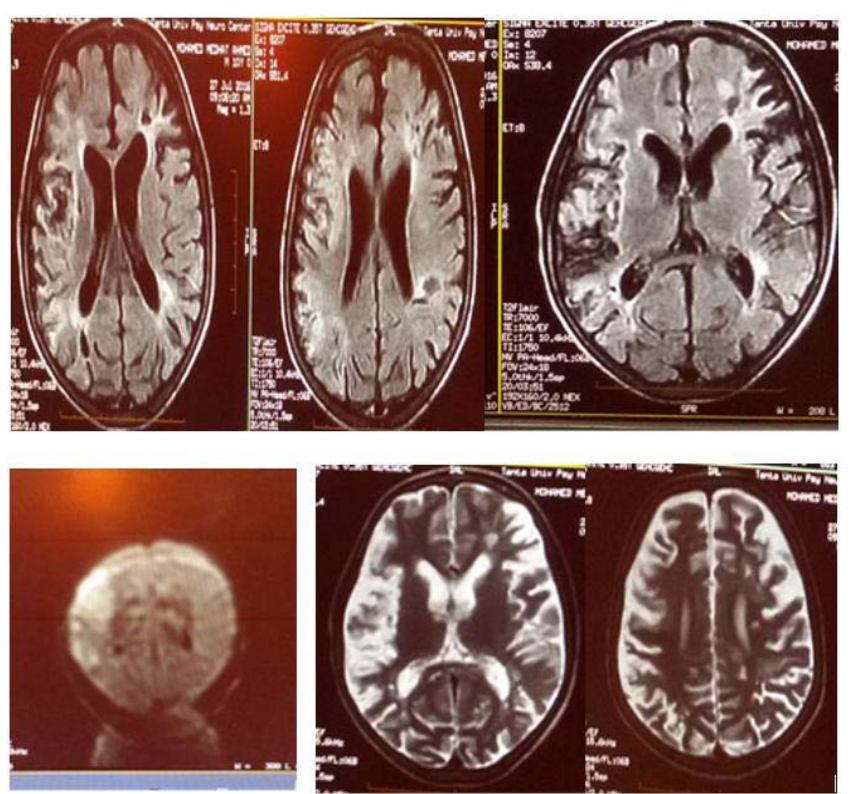

Figure (1b): MRI brain: upper panel showing axial flair demonstrating old right middle cerebral artery infarction besides multiple lacunar infarctions on the left side. Lower panel showing DWI and T2WI demonstrating right middle cerebral artery infarction besides multiple lacunar infarctions on the left side and marked atrophic changes in this case.

headache with migraine like character 3 years ago which became chronic 1 year later. Also, he experienced poor scholastic achievement (Stanford Binet IQ: 68) 2 years ago followed by repeated attacks of TIAs in the form of right side weakness one year ago followed 2 weeks later by 2 attacks of focal fits over right side of the body. There was past history of old left ischemic hemiplegia with dysarthria one and half years ago with partial improvement with residual deficit with history of post stroke seizures 1 month later on Levetiracetam, hydroxyurea, aspocid regular ttt, but irregular blood transfusion. He was scheduled for Hematopoietic Stem Cell Transplant (HSCT), but later after diagnosis with moyamoya syndrome 6 months ago with
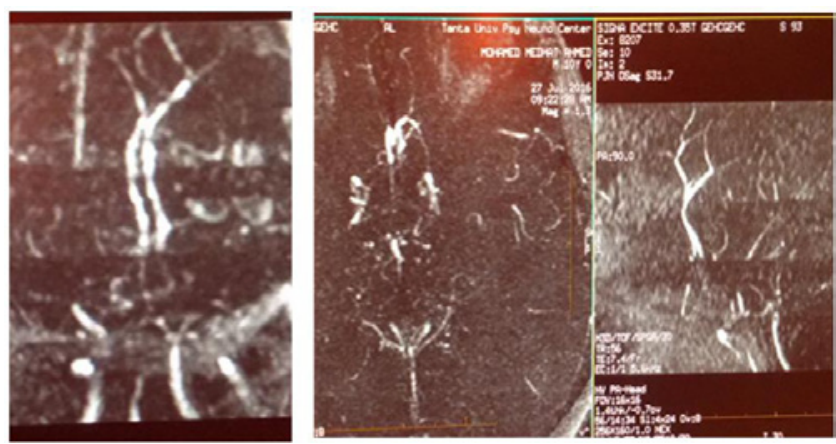

Figure (1c): MRA showing Moyamoya syndrome in this case

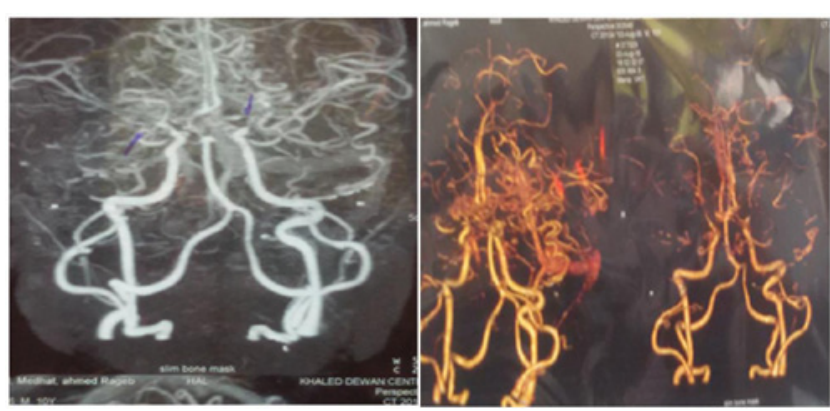

Figure (1d): CT angiography showing Moyamoya syndrome in this case.

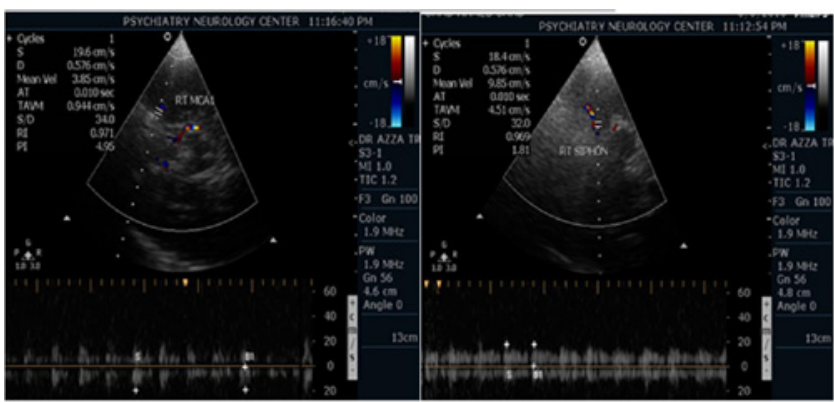

Figure (1e): TCCD showing TAMMV of different arteries less than $20 \mathrm{~cm} / \mathrm{s}$ (very low velocity) in this case.

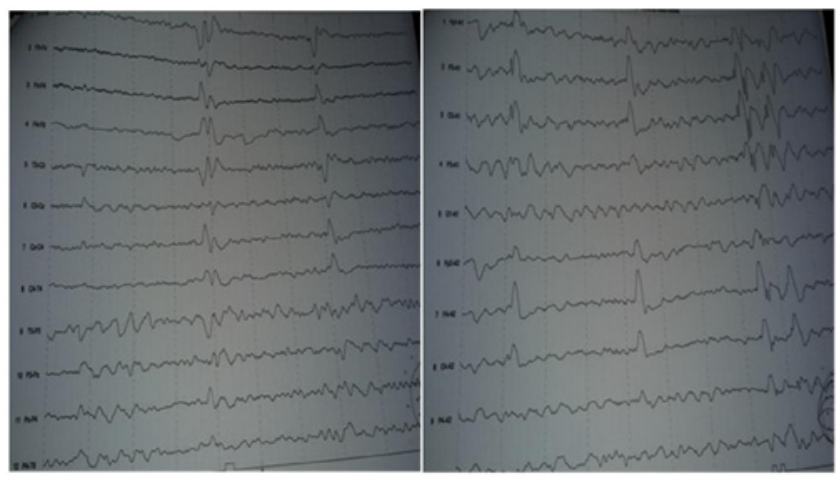

Figure (1f): Abnormal EEG showing multifocal epileptogenic activity in the form of spike, sharp and slow wave complex in this case.

MRI, MRA, TCCD and CT angiography, he was planned to undergo Encephaloduroarteriosynangiosis (EDAS). Serum levels of protein 
C and protein S were reduced (55 IU/dl for protein C, $45 \mathrm{IU} / \mathrm{dl}$ for protein S). We were still waiting for results of this intervention, which was postponed for covid-19 epidemic (Figures 1a-1f).

\section{Case 2}

A male child patient aged 7 years diagnosed at the age of 6 months with SCD with negative family history for SCD and positive consanguinity. He presented 3 years ago by recurrent attacks of right side weakness with mouth deviation to left side lasting for hours (TIA) with complete improvement after 5 days. One year later, he suffered from left side weakness associated with speech disturbance with patial improvement and residual weakness in lower limb. One month later, he developed recurrent attacks of left focal seizures with second generalization. He was controlled on regular treatment with Oxcarbazepine syrup. Patient also complained of recurrent episodic bitemporal headache with migraine- like character and his parents reported cognitive affection (Stanford Binet IQ: 54) I year ago. He had MRI, MRA, EEG, then patient had diagnostic catheter (endovascular) on brain with confirmation of diagnosis of moyamoya 2 years ago. The patient was on regular ttt with aspocid, cetal syrup for headache, hydroxyurea but infrequent blood transfusion. He was recommended to undergo direct STA-MCA surgery. Serum levels of protein $\mathrm{C}$ and protein $\mathrm{S}$ were reduced (59 IU/dl for protein C, $55 \mathrm{IU} / \mathrm{dl}$ for protein S) (Figures 2a-2f)

\section{Discussion}

Moyamoya disease was first described in Japan in 1957. Many similar cases have subsequently been reported, mainly in Japan and other Asian countries. The disease is found less frequently in North America and Europe [13]. Moyamoya syndrome is unilateral arterial constriction especially of the distal internal carotid artery occurs, or occurs when one of the several specified conditions is also present e.g. Down syndrome, sickle cell anemia, neurofibromatosis type 1, congenital heart disease, fibromuscular dysplasia, activated protein $\mathrm{C}$ resistance, or head trauma [14].

Moyamoya disease tends to affect adults in the third to fourth decade of life. In children it tends to cause strokes or seizures. In adults it tends to cause strokes or bleeding. The clinical features are strokes, recurrent Transient Ischemic Attacks (TIAs), sensorimotor paralysis (numbness and paralysis of the extremities), convulsions and/or migraine-like headaches. Moreover, following a stroke, secondary bleeding may occur. Such bleeding, called hemorrhagic strokes, may also stem from rupture of the weak neovascular vessel

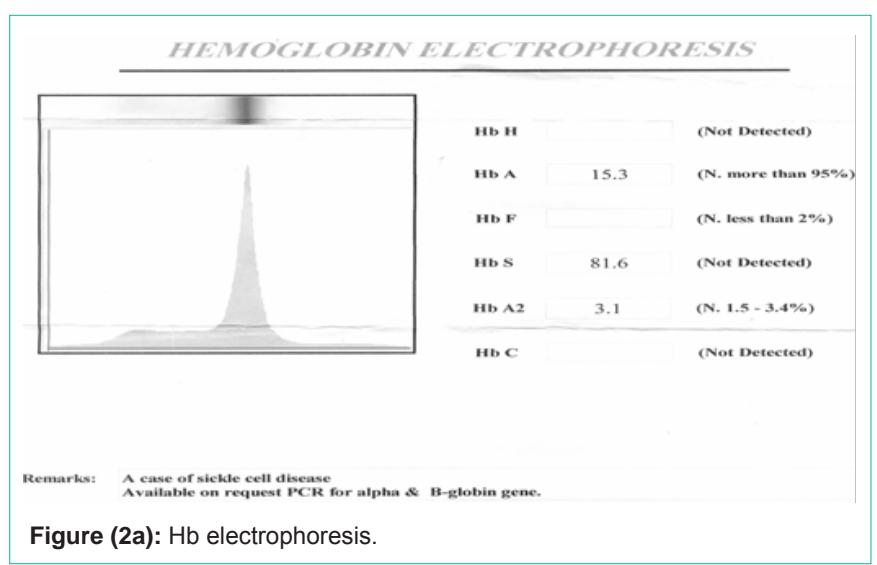

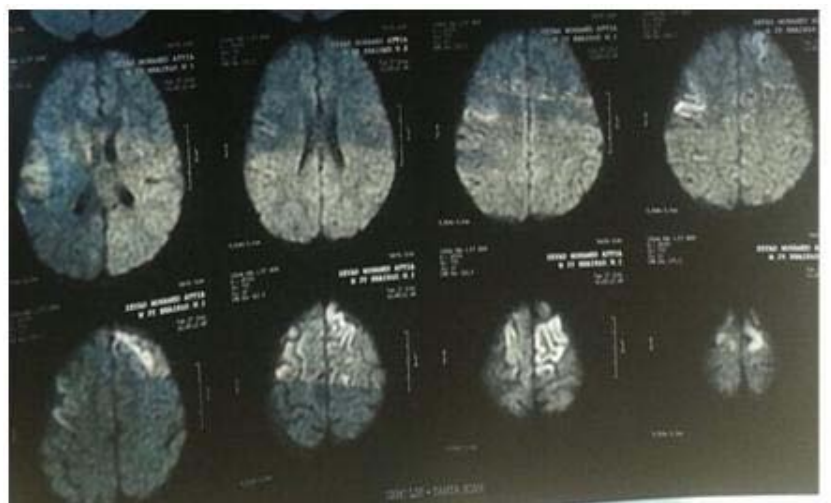

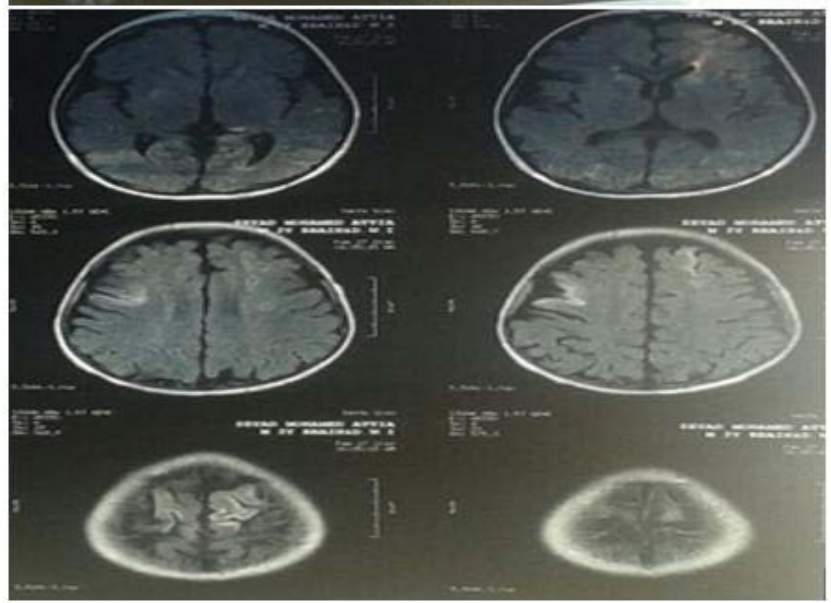

Figure (2b): MRI brain: upper panel showing DWI demonstrating multiple areas of restricted diffusion in left ACA and right MCA territories. Lower panel showing axial flair demonstrating right MCA and left ACA infarctions in this case.

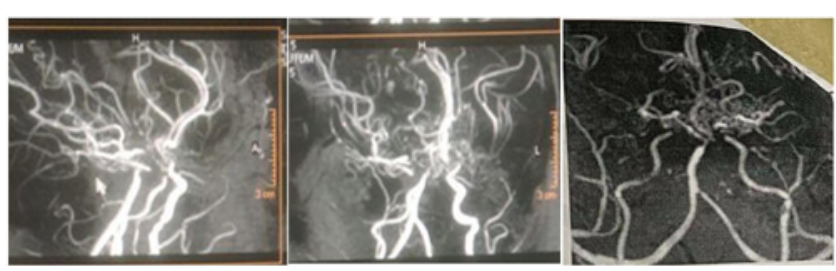

Figure (2c): Contrast enhanced MRA showing Moyamoya syndrome in this case.

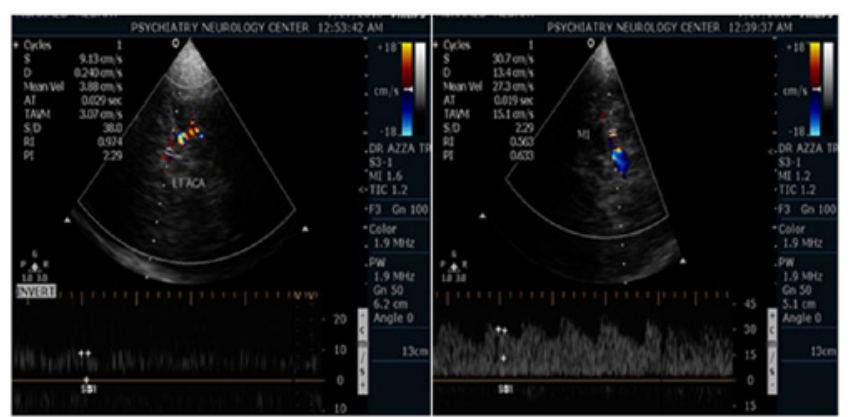

Figure (2d): 


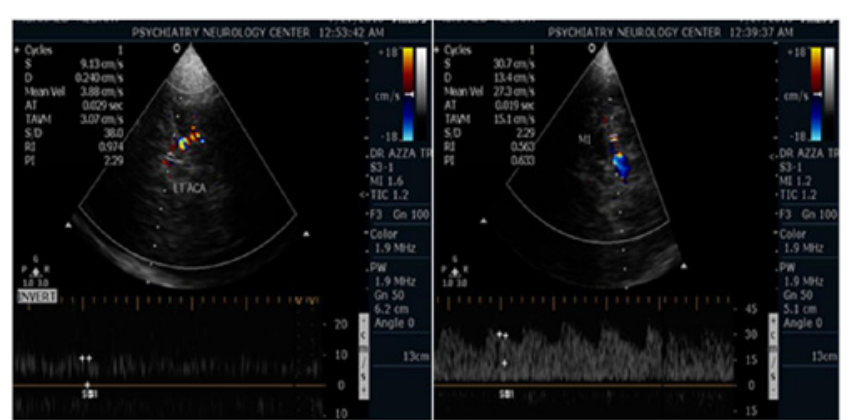

Figure (2e): TCCD showing TAMMV of different arteries less than $20 \mathrm{~cm} / \mathrm{s}$ (very low velocity) in this case.

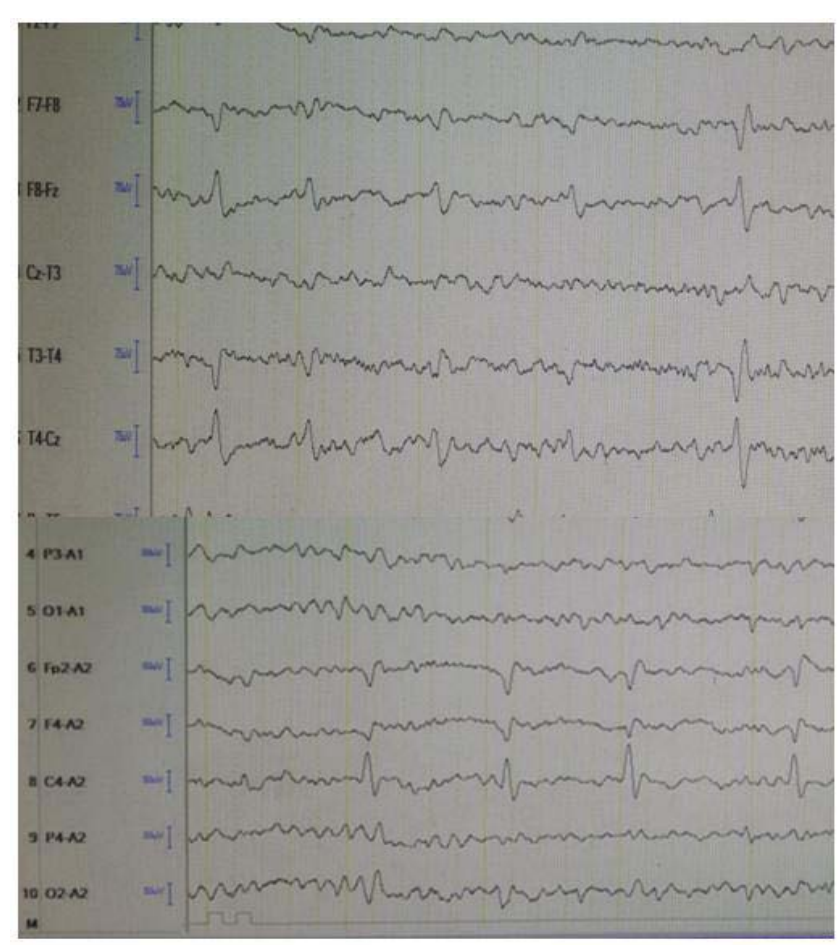

Figure (2f): Abnormal EEG showing multifocal epileptogenic activity in the form of spike, biphasic sharp and slow wave complex with phase reversal in this case.

walls [15].

Cerebral angiography is the gold standard of diagnosing Moyamoya disease and its progression. Magnetic Resonance Angiography (MRA) is also useful in diagnosing the disease with good correlation with Suzuki's grading system. Often nuclear medicine studies such as Single Photon Emission Computerized Tomography (SPECT) are used to demonstrate the decreased blood and oxygen supply to areas of the brain involved with moyamoya disease. Conventional angiography provides the conclusive diagnosis of moyamoya disease in most cases and should be performed before any surgical considerations [16].

SCD is a hypercoagulable state characterized by chronic activation of coagulation in vivo and increased risk of both arterial and venous thrombosis. There was increasing evidence that the activation of coagulation in SCD was not just a secondary event, but might contribute to disease pathogenesis. It was found that reduced plasma levels of physiologic anticoagulants was commonly observed in patients with SCD. This was explained by chronic consumption due to ongoing coagulation activation $[17,18]$.

Drugs such as antiplatelet agents (including aspirin) are usually given to prevent clots, but surgery is usually recommended. There are many operations that have been developed for the condition, but currently the most favored are the indirect procedures: Encephaloduroarteriosynangiosis (EDAS), Encephalomyosynangiosis (EMS), and multiple burr holes and the direct procedure STA-MCA. Direct Superficial Temporal Artery (STA) to Middle Cerebral Artery (MCA) bypass is considered the treatment of choice, although its efficacy, particularly for hemorrhagic disease, remains uncertain [19].

The long-term outlook for patients with treated moyamoya seems to be good when direct bypass is used. While symptoms may seem to improve almost immediately after the in-direct EDAS, EMS, and multiple burr holes surgeries, it will take probably 6-12 months before new vessels can develop to give a sufficient blood supply. With the direct STA-MCA surgery, increased blood supply is immediate [20].

\section{Conclusion}

Children with SCD who develop moyamoya syndrome are at significant risk of recurrent strokes and poor neurologic outcomes. The natural history of this disorder is not well known Patients with low stroke burden, or strokes limited to one hemisphere, may have a satisfactory long-term prognosis if further infarcts can be prevented. The ability to identify the predictive factors for the development of moyamoya in an at-risk population could lead to better outcomes for these patients through earlier diagnosis and treatment. Once a major stroke or bleeding takes place, even with treatment, the patient may be left with permanent loss of function so it is very important to treat this condition promptly.

\section{References}

1. Tanya G, Chen, Lathrop RP. The Case for Rapid Diagnosis of Sickle Cell Disease: A Literature Review. Journal of Global Health Perspective. 2012.

2. Patoka KP, Mark T. Gladwin: Vasculopathy and pulmonary hypertension in sickle cell disease. American Journal of Physiology - Lung Cellular and Molecular Physiology. 2015; 308: L314-L324.

3. Yawn BP, Buchanan GR, Afenyi-Annan AN, Ballas SK, Hassell KL, James $\mathrm{AH}$, et al. Management of sickle cell disease: summary of the 2014 evidencebased report by expert panel members. JAMA. 2014; 312: 1033-1048.

4. El-Beshlawy A, Youssry I. Prevention of hemoglobinopathy in Egypt. Hemoglobin. 2009; 1: S14-S20.

5. Hogan AM. Impact of frontal white matter lesions on performance monitoring: ERP evidence for cortical disconnection. Brain. 2006; 129: 2177-2188.

6. Dobson SR, Holden KR, Nietert PJ, et al. Moyamoya syndrome in childhood sickle cell disease: a predictive factor for recurrent cerebrovascular events. Blood. 2002; 99: 3144-3150.

7. Scott RM, Smith ER. Moyamoya disease and moyamoya syndrome. N Engl J Med. 2009; 360: 1226-1237.

8. Oka K, Yamashita M, Sadoshima S, Tanaka K. Cerebral haemorrhage in Moyamoya disease at autopsy. Virchows Arch A Pathol Anat Histol. 1981; 392: $247-261$.

9. Yamashita M, Tanaka K, Matsuo T, Yokoyama K, Fujji T, Sakamoto H. Cerebral dissecting aneurysms in patients with moyamoya disease: report of 
two cases. J Neurosurg. 1983; 58: 120-125.

10. Jauch EC, Jeffrey L. Saver: Guidelines for the Early Management of Patients With Acute Ischemic Stroke. AHA/ASA Guideline. 2013; 44: 870-947.

11. Al-Jafar H, Hashem K, Alhasan AA, Lamdhade S, AlDallal S, Alhaji FA, et al. Moyamoya Disease: A Rare Sickle Cell Trait Neurological Complication. J Neurol Psychol. 2016; 4: 3

12. Shoukat S, Itrat A, Taqui AM, Zaidi M, Kamal AK. Moyamoya disease: A clinical spectrum, literature review and case series from a tertiary care hospital in Pakistan. BMC Neurol. 2009; 9: 15

13. Suzuki J, Kodama N. Moyamoya disease--a review. Stroke 1983; 14: 104109.

14. Ganesan V, Smith ER. "Moyamoya: Defining current knowledge gaps". Developmental Medicine \&Child Neurology. 2015; 57: 786-787.

15. Hishikawa T, Sugiu K, Date I. "Moyamoya Disease: A Review of Clinical Research". 2016
16. Janda P, Bellew JG, Veerappan V. "Moyamoya disease: case report and literature review". The Journal of the American Osteopathic Association. 2009; 109: 547-553.

17. Ataga $\mathrm{KI}$, Brittain JE, Desai $\mathrm{P}$. Association of coagulation activation with clinical complications in sickle cell disease. PLoS One. 2012; 7: e29786.

18. Bayazit AK, Kilinc Y. Natural coagulation inhibitors (protein C, protein S, antithrombin) in patients with sickle cell anemia in a steady state. Pediatr Int off J Jpn Pediatr Soc. 2001; 43: 592-596.

19. Smith ER. "Moyamoya Arteriopathy". Current Treatment Options in Neurology. 2012; 14: 549-556

20. Sun H, Wilson C, Ozpinar A, Safavi-abbasi S, Zhao Y, Nakaji P, et al. "Perioperative Complications and Long-Term Outcomes after Bypasses in Adults with Moyamoya Disease: A Systematic Review and Meta-Analysis". World Neurosurgery. 2016; 92: 179-188. 